\title{
A comparison between exposure-response relationships for wind turbine annoyance and annoyance due to other noise sources
}

\author{
Sabine A. Janssen ${ }^{\text {a) }}$ and Henk Vos \\ Department of Urban Environment and Safety, Netherlands Organization for Applied Scientific Research, \\ P.O. Box 49, 2600 AA Delft, The Netherlands \\ Arno R. Eisses \\ Department of Acoustics and Sonar, Netherlands Organization for Applied Scientific Research, \\ P.O. Box 96864, 2509 JG The Hague, The Netherlands \\ Eja Pedersen ${ }^{\text {b) }}$ \\ Ecology and Environmental Science, Halmstad University, P.O. Box 823, SE-301 18 Halmstad, Sweden
}

(Received 4 November 2010; revised 17 June 2011; accepted 28 September 2011)

\begin{abstract}
Surveys have shown that noise from wind turbines is perceived as annoying by a proportion of residents living in their vicinity, apparently at much lower noise levels than those inducing annoyance due to other environmental sources. The aim of the present study was to derive the exposureresponse relationship between wind turbine noise exposure in $L_{\text {den }}$ and the expected percentage annoyed residents and to compare it to previously established relationships for industrial noise and transportation noise. In addition, the influence of several individual and situational factors was assessed. On the basis of available data from two surveys in Sweden $(N=341, N=754)$ and one survey in the Netherlands $(N=725)$, a relationship was derived for annoyance indoors and for annoyance outdoors at the dwelling. In comparison to other sources of environmental noise, annoyance due to wind turbine noise was found at relatively low noise exposure levels. Furthermore, annoyance was lower among residents who received economical benefit from wind turbines and higher among residents for whom the wind turbine was visible from the dwelling. Age and noise sensitivity had similar effects on annoyance to those found in research on annoyance by other sources. () 2011 Acoustical Society of America. [DOI: 10.1121/1.3653984]
\end{abstract}

PACS number(s): 43.50.Qp, 43.50.Rq [BSF]

Pages: $3746-3753$

\section{INTRODUCTION}

Community noise is a recognized problem that appears to be increasing, due to both increased noise exposure from existing sources such as road traffic and to the implementation of new noise sources such as wind turbines. While wind turbines have beneficial effects for the environment because they offer a clean substitute for fossil fuels, they inevitably generate environmental noise. Recent studies investigating the community response to wind turbine noise have shown that a proportion of the residents living in the vicinity of wind turbines perceive the noise generated by them as being annoying (van den Berg et al., 2008; Pedersen et al., 2009). Noise generated by the rotor blades is perceived as the most annoying, although other aspects, such as the alteration of the landscape and the perception of rotor blade movement, are often considered annoying as well. Visual factors (e.g., being able to see one or more wind turbines from the dwelling) and environmental factors (e.g., living in rural versus built-up areas) have been found to influence the degree of perceived annoyance (Pedersen and Larsman, 2008). For example, wind turbine noise-induced annoyance occurs more often when wind turbines are visible from the dwelling

\footnotetext{
${ }^{\text {a) }}$ Author to whom correspondence should be addressed. Electronic mail: sabine.janssen@tno.nl

b) Also at Department of Occupational and Environmental Medicine, University of Gothenburg, P.O. Box 414, 40530 Gothenburg, Sweden.
}

or when the presence of wind turbines is perceived to have a negative impact upon the landscape. Although the degree of urbanization has been found to moderate wind turbine noiseinduced annoyance, the nature of this influence is unclear: In Sweden annoyance was highest in rural areas, whereas in the Netherlands, the highest annoyance was reported in built-up areas. Furthermore, in the Netherlands, people that benefitted economically from the implementation of wind turbines reported almost no annoyance, even though on average their exposure to wind turbine noise is higher. Besides its reported impact on annoyance, wind turbine noise exposure has, at higher noise levels, been found to disturb sleep as well (van den Berg et al., 2008; Pedersen, 2011). Moreover, respondents who reported being annoyed by wind turbine noise also reported experiencing more symptoms relating to stress, feeling less well rested in the morning, and consider their environment as less suitable to rest and psychophysiological restoration (Pedersen and Persson Waye, 2007, 2008).

Findings suggest that at equal noise exposure levels, the expected annoyance due to wind turbine noise might be higher than annoyance due to other environmental noise sources (Pederson and Persson Waye, 2004; van den Berg, 2008; Pedersen et al., 2009). The annoyance also appears to be high in comparison to exposure-response relationships for stationary sources, suggesting that wind turbines should be treated as a new type of source. However, there are several reasons why the observed relationships between wind 
turbine noise and annoyance cannot be compared directly to the earlier established exposure-response relationships for transportation noise (Miedema and Vos, 1998; Miedema and Oudshoorn, 2001) or for industrial noise from stationary sources (Miedema and Vos, 2004). First, exposure-response relationships for wind turbine noise were derived using noise exposure measures that do not correspond to international standards for assessing the impact of community noise $\left(L_{d e n}\right.$ or $L_{d n}$ ). Second, exposure-response relationships for wind turbine noise were derived for annoyance perceived outdoors at the dwelling, while established exposure-response relationships for other noise sources typically do not distinguish between annoyance indoors or outdoors. Third, different methods have been used to quantify the exposure-response relationships.

In the present study, exposure-response relationships between the exposure metric $L_{d e n}$ and self-reported annoyance indoors as well as outdoors due to wind turbines were derived using the method previously used to derive the exposure-response relationships for transportation and industrial noise. In addition, the influence of several individual and situational factors was assessed, such as age, noise sensitivity, economical benefit, and visibility of the wind turbine. The analysis was done on available data that were collected during previous studies in Sweden and the Netherlands. The newly derived relationship was compared to earlier established exposure-response relationships for industrial noise from stationary sources and transportation noise.

\section{METHOD}

\section{A. Study design and study sample}

Data from two studies conducted in Sweden (2000 and 2005) and one study in the Netherlands (2007) were used. Both Swedish studies were conducted during the summer and had cross-sectional designs with a sample of respondents who were exposed to varying levels of wind turbine noise. The 2000 study (Pedersen and Persson Waye, 2004) was conducted in the south of Sweden in an area characterized primarily by agriculture in an overall flat even landscape. The 2005 Swedish study (Pedersen and Persson Waye, 2007) was conducted in areas characterized by different types of terrain (i.e., even/flat versus complex) and varying degrees of urbanization (i.e., rural versus built-up). In both studies, questionnaires were used. Of the 513 questionnaires delivered to residents in the 2000 study, 351 (68\%) usable questionnaires were returned. In the 2005 study, 1309 questionnaires were sent to residents; 754 (58\%) returned questionnaires were usable questionnaires. Respondents did not differ from the population in the study areas in distribution of age or gender nor did late responders diverge from early responders in their answers, suggesting that the respondents represented the study population satisfactorily.

The study in the Netherlands (van den Berg et al., 2008; Pedersen et al., 2009) included a sample of the population living within a $2.5 \mathrm{~km}$ radius of a wind turbine, stratified according to wind turbine immission levels [sound pressure levels $<30,30-35,35-40,40-45,>45 \mathrm{~dB}(\mathrm{~A})]$ and environment type (rural/quiet, rural with main roads, built-up). At a response rate of at least 30\%, a minimum of 50 respondents per stratum $(4 \times 3=12$ strata $)$ was envisaged. A postal questionnaire, based on the Swedish questionnaire, was sent during April 2007. Of the 1948 questionnaire posted, 725 (37\%) usable questionnaires were returned. All respondents received a gift voucher. A short questionnaire comprising two key-questions about annoyance outdoors and indoors was sent to a random sample of 200 non-respondents of which 95 (48\%) responded. No differences in reported level of annoyance were found between the original respondents and these non-respondents.

\section{B. Noise exposure}

Annual day-evening-night A-weighted equivalent noise level $\left(L_{d e n}\right)$ was defined in accordance with EU environmental noise guidelines. $L_{\text {den }}$ was calculated from the immission levels determined in the original studies [see Pedersen and Persson Waye $(2004,2007)$ and van den Berg et al. (2008), respectively]. For each respondent, outdoor A-weighted sound pressure levels from the nearest wind turbine(s) were determined for a neutral atmosphere at a constant wind velocity of $8 \mathrm{~m} / \mathrm{s}$ at a height of $10 \mathrm{~m}$ in the direction towards the respondent, which is the reference wind velocity by convention (e.g., Swedish Environmental Protection Agency, 2001). To these data, a correction of $+4.7 \mathrm{~dB}(\mathrm{~A})$ was applied, calculated by van den Berg (2008) as the mean difference between $L_{d e n}$ and the A-weighted sound pressure level as specified above at a given distance from a wind turbine. While in principle the correction depends on the wind velocity distribution at a specific location, the type of wind turbine and the hub height, statistical wind velocity data was not available for all study locations. Furthermore, using a variable correction factor for the situation in the Netherlands did not provide a better prediction of annoyance in comparison to $L_{d e n}$ calculated with the fixed correction factor.

\section{Questionnaire}

In all three studies, annoyance due to wind turbines and other environmental stressors were assessed with the following question: "The list below summarizes a number of aspects that you may be aware of and/or be annoyed by when you spend time indoors at your dwelling. Please indicate for each aspect whether you are aware of it and whether it annoys you." The response to each aspect was registered on a five-point scale: $1=$ "do not notice," $2=$ "notice, but not annoyed," 3 = "slightly annoyed," 4 = "rather annoyed," and $5=$ "very annoyed." The same question was asked for annoyance outdoors at the the dwelling. Visibility of the wind turbine from within the home was assessed by the question: "Can you see a wind turbine from your dwelling or your garden/balcony?" to which the responses "yes" or "no" was possible. To assess whether respondents benefitted economically from wind turbines, the questionnaire posed the question "Are you a (co) owner of one or more wind turbines?" to which the answers "yes" or "no" could be given. Noise sensitivity was assessed along a 4-point scale, from 1 "not sensitive" to 4 "very sensitive" except for the study in the Netherlands in which noise sensitivity was 
TABLE I. Individual and situational characteristics, plus percentages for each annoyance category indoors and outdoors, per study and in total.

\begin{tabular}{|c|c|c|c|c|c|c|c|c|}
\hline & \multicolumn{2}{|c|}{ Sweden 2000} & \multicolumn{2}{|c|}{ Sweden 2005} & \multicolumn{2}{|c|}{ Netherlands 2007} & \multicolumn{2}{|c|}{ Total } \\
\hline & \multicolumn{2}{|c|}{$n=341$} & \multicolumn{2}{|c|}{$n=754$} & \multicolumn{2}{|c|}{$n=725$} & \multicolumn{2}{|c|}{$n=1820$} \\
\hline & Mean or $\%$ & SD & Mean or $\%$ & $\mathrm{SD}$ & Mean or $\%$ & SD & Mean or $\%$ & $\mathrm{SD}$ \\
\hline$L_{d e n}$ & 39.3 & 3.2 & 38.1 & 3.1 & 39.8 & 6.4 & 39.0 & 4.8 \\
\hline Age & 47.2 & 14.0 & 50.9 & 15.0 & 54.3 & 15.0 & 51.5 & 15.0 \\
\hline Noise sensitivity & 51.0 & 20.9 & 50.7 & 22.3 & 46.1 & 23.8 & 48.9 & 22.7 \\
\hline Female $(\%)$ & 58.5 & & 55.6 & & 49.2 & & 53.6 & \\
\hline Economic benefit (\%) & 3.0 & & 2.7 & & 14.3 & & 7.6 & \\
\hline Visible $(\%)$ & 94.4 & & 70.6 & & 67.8 & & 74.0 & \\
\hline Rural (\%) & 40.2 & & 24.5 & & 70.5 & & 45.8 & \\
\hline Flat terrain $(\%)$ & 100.0 & & 50.3 & & 100.0 & & 79.4 & \\
\hline Annoyance 0-100 & Indoors & Outdoors & Indoors & Outdoors & Indoors & Outdoors & Indoors & Outdoors \\
\hline $0-25(\%)$ & 88.5 & 66.9 & 96.4 & 88.6 & 86.4 & 76.7 & 91.0 & 79.8 \\
\hline $25-50(\%)$ & 4.1 & 17.6 & 2.4 & 7.3 & 7.7 & 13.0 & 4.8 & 11.5 \\
\hline $50-75(\%)$ & 4.1 & 6.5 & 1.1 & 2.3 & 3.0 & 6.2 & 2.4 & 4.6 \\
\hline $75-100(\%)$ & 3.2 & 9.1 & 0.1 & 1.9 & 2.9 & 4.1 & 1.8 & 4.1 \\
\hline
\end{tabular}

assessed along a five-point scale, ranging from $1=$ "not sensitive" to $5=$ "extremely sensitive." Furthermore, the questionnaire contained questions concerning demographic characteristics, health, and the attitude regarding wind turbines.

In the present study, the five-point scale was recoded to a four-point scale: Categories 1 and 2 were combined to obtain a new category $1=$ "not annoyed." Subsequently, the annoyance as well as the noise sensitivity response categories were converted into scales ranging from 0 to 100 . This conversion is based on the assumption that a set of categories divides the range of 0 to 100 in equally spaced intervals. The general rule that gives the position of an inner category boundary on the scale of 0 to 100 is: score boundary $i_{i}=100 \cdot \mathrm{i} / \mathrm{m}$, where $i$ is the rank number of the category boundary, starting from 1 for the upper boundary of the lowest category, and $m$ is the number of categories. The percentage of responses exceeding a certain cut-off point on the scale may be reported. Following convention, if the cut-off is 72 on a 0-100 scale, the result is called the percentage of "highly annoyed" persons (\%HA). Likewise, a cut-off of 50 indicates the percentage of "annoyed" persons $(\% A)$.

\section{Statistical model}

The statistical model described by Miedema and Oudshoorn (2001), Groothuis-Oudshoorn and Miedema (2006) was employed here. This model states that the percentage annoyed persons at a given value of $L_{d e n}$ is:

$P_{C}\left(L_{d e n}\right)=100 \cdot\left(1-\Phi\left(\left(C-\left[\beta_{0 \mathrm{i}}+\beta_{1} L_{d e n}+\sum_{\mathrm{i}} \beta_{\mathrm{i}} X_{\mathrm{i}}\right]\right) / \sigma\right)\right)$,

where $P_{C}\left(L_{d e n}\right)$ represents the percentage of persons exposed to $L_{d e n}$ with an annoyance score above the cut-off point $C$ (i.e., 72 for $\% H A, 50$ for $\% A$ ), $\Phi$ represents the cumulative standard normal distribution. Parameter $\beta_{0}$ is the model intercept, whereas $\beta_{1}$ is the gradient that describes the change in self-reported annoyance on the 100-point scale as a function of $L_{d e n} . X_{i}$ and $\beta_{\mathrm{i}}$ represent additional predictors and their corresponding parameters within the model describe the change in self-reported annoyance as a function of these variables. The distributions of the error components were assumed to be normal with a mean equal to zero.

By applying this statistical model, a basic model of selfreported annoyance was derived for the combined data from Sweden and the Netherlands. Data from the Swedish studies were distinguished from the data from the Netherlands (i.e., the reference) by separate dummy variables (Swe00 and Swe05; equal to 1 if data is from respective Swedish study, equal to zero otherwise). The gradient of the effect of $L_{d e n}$ on annoyance $\left(\beta_{1}\right)$ was assumed to be constant between studies. Next, the following extra variables $\left(X_{i}\right)$ were added to the basic model: age and $a g e^{2}$ (age squared added because annoyance and age have previously been found to show an inverse U-shaped relationship), gender, noise sensitivity, economic benefit, visibility, degree of urbanization [i.e., rural versus built-up: (1) for the Netherlands defined on the basis of the environmental address density; (2) for the Swedish data as defined during the original studies], and terrain (i.e., even/flat versus complex). Using a stepwise backward elimination procedure, at each step, the variable with the least significant contribution (i.e., highest $p$-value) was removed, until all variables contributed with $P \leq 0.05$. Last, a new model without any extra variables $\left(X_{i}\right)$ or dummy variables (Swe00 and Swe05) was set up, resulting in a single exposure-response relationship for annoyance and $L_{d e n}$ with a fixed intercept $\left(\beta_{0}\right)$ and fixed gradient $\left(\beta_{1}\right)$ for the three studies combined.

\section{RESULTS}

\section{A. General}

Categories 1 and 2 of the five-point annoyance scale were combined as described in the preceding text (i.e., "not annoyed") to give a converted four-point annoyance scale. 
TABLE II. Results of the annoyance indoors basic model (far left column) and backward model (far right column). Dummy variables Swe00 and Swe05 equal to 1 indicate data from 2000 and 2005 Swedish studies, respectively. Statistically significant effects $(P<0.05)$ are underlined.

\begin{tabular}{|c|c|c|c|c|c|c|c|c|c|}
\hline & Basic & Age & Female & Sensitive & Eco. benefit & Visible & Rural & Flat terrain & Backward \\
\hline$\beta_{0}$ & $-\underline{154.40}$ & $-\underline{213.01}$ & $-\underline{145.94}$ & $-\underline{180.07}$ & $-\underline{201.12}$ & $-\underline{142.71}$ & $-\underline{155.26}$ & $-\underline{152.06}$ & $-\underline{242.88}$ \\
\hline$L_{d e n}$ & $\underline{3.08}$ & $\underline{3.25}$ & $\underline{3.11}$ & $\underline{3.03}$ & $\underline{4.47}$ & $\underline{2.21}$ & $\underline{3.01}$ & $\underline{3.09}$ & $\underline{3.65}$ \\
\hline Swe00 & 2.16 & 3.35 & 2.10 & $\overline{0.43}$ & -3.12 & -4.91 & 3.59 & 2.19 & $-\underline{9.03}$ \\
\hline Swe05 & -24.90 & -24.44 & -25.37 & -27.46 & $-\underline{28.08}$ & $-\underline{26.95}$ & -22.95 & $-\underline{26.23}$ & $-3 \overline{1.27}$ \\
\hline Age & & $\underline{166.38}$ & & & & & & & $\overline{44.26}$ \\
\hline $\operatorname{Age}^{2}$ & & $-\overline{119.78}$ & & & & & & & \\
\hline Female & & & -6.33 & & & & & & \\
\hline Sensitive & & & & 0.60 & & & & & $\underline{0.56}$ \\
\hline Economic benefit & & & & & $-\underline{64.00}$ & & & & $-\underline{56.74}$ \\
\hline Visible & & & & & & $\underline{33.38}$ & & & $\overline{33.70}$ \\
\hline Rural & & & & & & & 5.09 & & \\
\hline Flat terrain & & & & & & & & -2.93 & \\
\hline
\end{tabular}

The mid-points of this converted scale along a 100-point scale are $12.5,37.5,62.5$, and 87.5. Table I provides an overview of the individual and situational characteristics, plus the annoyance scores (indoors and outdoors) along the 100point scale, for each of the three studies and in total.

The highest wind turbine noise exposure levels $\left(L_{d e n}\right)$ were encountered in the study in the Netherlands. The majority of Swedish respondents were exposed to $L_{d e n}$ levels between 35 and $40 \mathrm{~dB}$, while a relatively large proportion of respondents in the Netherlands were exposed to $L_{d e n}$ levels below $35 \mathrm{~dB}$ and levels over $45 \mathrm{~dB}$. This may partly be attributed to differences in study design: in the Netherlands the stratification was based on noise exposure levels, whereas in Sweden locations were selected mainly on the basis of geographical areas.

\section{B. Study differences and the role of individual and situational characteristics}

Table II provides an overview of the basic model of annoyance indoors (far left column), and the model following the backward elimination procedure (far right column). The central columns depict the basic annoyance model plus a single extra variable per column. In all models, $L_{\text {den }}$ is positively related to annoyance indoors, with an expected increase of around 3 on the 100-point annoyance scale per $1 \mathrm{~dB}$ increase in $L_{d e n}$. In addition, several individual and situational variables are found to influence annoyance: annoyance is found to decrease with economical benefit and to increase with noise sensitivity, visibility, and age (however, there is a tendency for a curvilinear effect with highest annoyance in the middle-aged group). The dummy variables Swe00 and Swe05 indicate that annoyance indoors is lower in the 2005 Swedish study (and slightly lower in the 2000 study) than in the study in the Netherlands, even more so when adjusted for other variables.

Table III provides an overview of the basic model of annoyance outdoors (far left column), and the model following the backward elimination procedure (far right column). The central columns depict the basic annoyance model plus a single extra variable per column. In all models, $L_{d e n}$ is positively related to annoyance outdoors, with an expected increase of 3 or more on the 100-point annoyance scale per 1 $\mathrm{dB}$ increase in $L_{\text {den }}$. Again, age, noise sensitivity, economical benefit, and visibility are found to influence annoyance outdoors, although here the curvilinear effect of age is significant. The dummy variables SweO0 and Swe05 indicate that annoyance outdoors is lower in the 2005 Swedish study than

TABLE III. Results of the annoyance outdoors basic model (far left column) and backward model (far right column). Dummy variables Swe00 and Swe05 equal to 1 indicate data from 2000 and 2005 Swedish studies respectively. Statistically significant effects $(P<0.05)$ are underlined.

\begin{tabular}{|c|c|c|c|c|c|c|c|c|c|}
\hline & Basic & Age & Female & Sensitive & Eco. benefit & Visible & Rural & Flat terrain & Backward \\
\hline$\beta_{0}$ & -148.05 & -197.96 & -146.09 & -170.36 & -193.80 & -138.92 & -149.20 & -147.83 & -215.34 \\
\hline$L_{d e n}$ & $\underline{3.36}$ & 3.36 & 3.36 & 3.30 & $\underline{4.74}$ & $\underline{2.52}$ & $\underline{3.30}$ & $\underline{3.36}$ & 3.85 \\
\hline Swe00 & $\overline{19.91}$ & 20.29 & $\overline{19.91}$ & $1 \overline{7.85}$ & $1 \overline{4.20}$ & $1 \overline{2.76}$ & $2 \overline{1.31}$ & $\overline{19.92}$ & $\overline{7.57}$ \\
\hline Swe05 & $-\overline{13.44}$ & $-\overline{13.12}$ & $-\overline{13.48}$ & $-\overline{15.58}$ & $-\overline{16.16}$ & $-\overline{14.82}$ & $-\overline{11.46}$ & $-\overline{13.57}$ & $-\underline{17.79}$ \\
\hline Age & & $1 \overline{86.07}$ & & & & $\overline{33.31}$ & & & $\overline{24.96}$ \\
\hline $\mathrm{Age}^{2}$ & & -160.24 & & & & & & & \\
\hline Female & & & -1.35 & & & & & & \\
\hline Sensitive & & & & 0.54 & & & & & 0.47 \\
\hline Economic benefit & & & & & -62.14 & & & & $-5 \overline{5.86}$ \\
\hline Visible & & & & & & 33.31 & & & $\overline{29.42}$ \\
\hline Rural & & & & & & & 4.99 & & \\
\hline Flat terrain & & & & & & & & -0.29 & \\
\hline
\end{tabular}


TABLE IV. Model of the exposure-response relationships between $L_{\text {den }}$ and annoyance indoors and outdoors. Significant effects $(P<0.05)$ are underlined.

\begin{tabular}{|c|c|c|c|c|}
\hline \multirow[b]{2}{*}{ Parameter } & \multicolumn{2}{|c|}{ Annoyance indoors } & \multicolumn{2}{|c|}{ Annoyance outdoors } \\
\hline & Coefficient & SE & Coefficient & SE \\
\hline$\beta_{0}$ & $-\underline{255.06}$ & 20.71 & $-\underline{226.88}$ & 19.21 \\
\hline \multirow[t]{3}{*}{$L_{d e n}$} & $\underline{5.50}$ & 0.50 & $\underline{5.48}$ & 0.45 \\
\hline & \multicolumn{2}{|c|}{ Covariance matrix } & & \\
\hline & $\beta_{0}$ & $L_{d e n}$ & $\beta_{0}$ & $L_{d e n}$ \\
\hline$\beta_{0}$ & 428.86 & -10.13 & 369.09 & -8.64 \\
\hline$L_{\text {den }}$ & -10.13 & 0.25 & -8.64 & 0.21 \\
\hline
\end{tabular}

in the study in the Netherlands, while there was no significant difference between the 2000 Swedish study and the study in the Netherlands when adjusted for other variables.

\section{Exposure-response relationships for annoyance indoors and outdoors}

For the modeling of a community exposure-response relationship, some considerations had to be taken. Because respondents with economical benefit hardly reported any annoyance despite living primarily in the highest exposure categories, including this relatively small number of residents would contaminate the relationship. Furthermore, due to oversampling in the highest exposure categories in the study in the Netherlands, the number of individuals who benefitted economically from the use of wind turbines has been exaggerated in the present sample. Therefore, in line with van den Berg et al. (2008), exposure-response relationships were derived only for respondents who did not benefit economically from wind turbines. Also, the relationships were derived without taking the study effect (i.e., Swe00 and Swe05) into account. The resulting model of the exposureresponse relationships is shown in Table IV. Note that the exclusion of individuals with economic benefit results in a

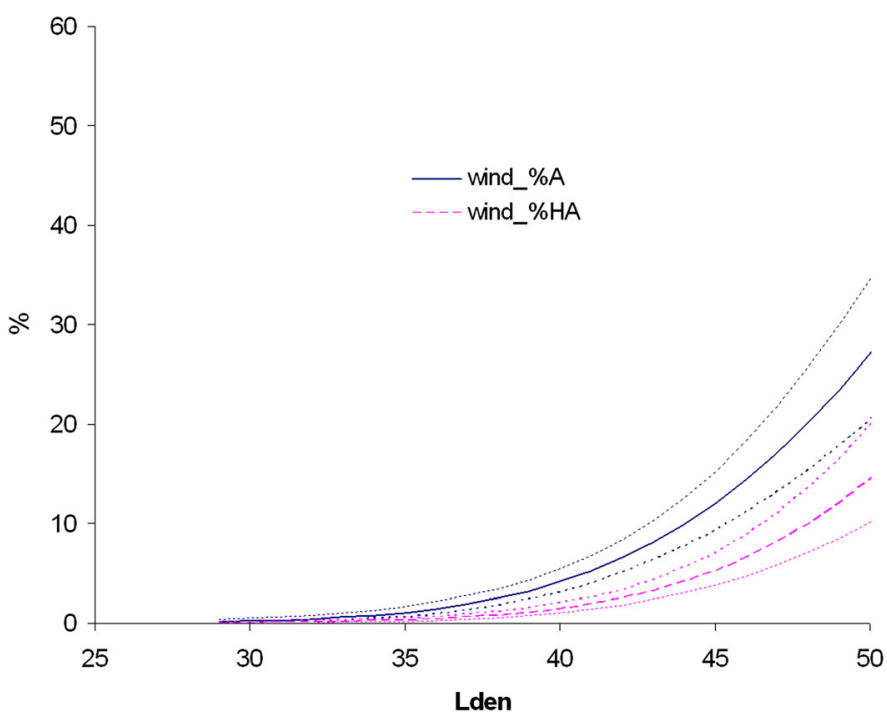

steeper slope of the relationship between exposure and annoyance than in the models presented in Tables II and III.

For practical purposes, the following polynomials were fitted to the exposure-response model across an $L_{d e n}$ range of 29-50 dB to approximate the percentage annoyed persons $(\% A)$ and the percentage highly annoyed persons (\%HA) indoors at a given noise exposure. Within the fitted noise exposure ranges, the values estimated by the polynomials closely match the values calculated with the model based on the data.

$$
\begin{aligned}
\% A_{\text {indoors }}= & -95.68+9.277 L_{\text {den }}-0.302 L_{\text {den }}^{2} \\
& +0.003313 L_{\text {den }}^{3}, \\
\% H A_{\text {indoors }}= & -107.60+9.656 L_{\text {den }}-0.289 L_{\text {den }}^{2} \\
& +0.002894 L_{\text {den }}^{3}, \\
\% A_{\text {outdoors }}= & 34.25-0.8464 L_{\text {den }}-0.0548 L_{\text {den }}^{2} \\
& +0.001551 L_{\text {den }}^{3}, \\
\% H A_{\text {outdoors }}= & -97.94+9.627 L_{\text {den }}-0.3175 L_{\text {den }}^{2} \\
& +0.003522 L_{\text {den }}^{3} .
\end{aligned}
$$

In Fig. 1, the exposure-response relationships and their 95\% confidence intervals are displayed up to a maximum of 50 $\mathrm{dB}$ because relatively few respondents were exposed to noise beyond this level, and extrapolation to higher exposure categories leads to increased uncertainty. According to the model, $L_{d e n}$ levels between 30 and $35 \mathrm{~dB}$ cause almost no annoyance, while an $L_{d e n}$ level of $45 \mathrm{~dB}$ corresponds to $12 \%$ and $26 \%$ annoyed persons indoors and outdoors, respectively. Note that the percentages of annoyed persons indoors are approximately the same as the percentages of highly annoyed persons outdoors.

\section{Comparison to exposure-response relationships for industrial and transportation noise}

Figures 2 and 3 provide a comparison of the exposureresponse relationships for the percentage annoyed $(\% A)$ and

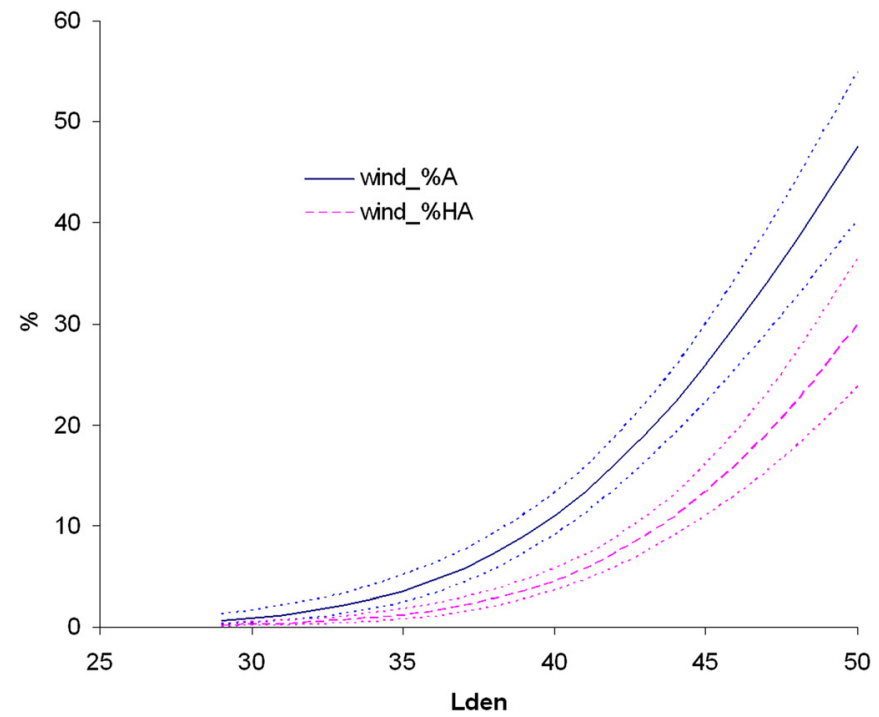

FIG. 1. (Color online) The exposure-response relationships between $L_{d e n}$ and the percentage of residents annoyed (\%A) and highly annoyed (\%HA) indoors (left) and outdoors (right). 

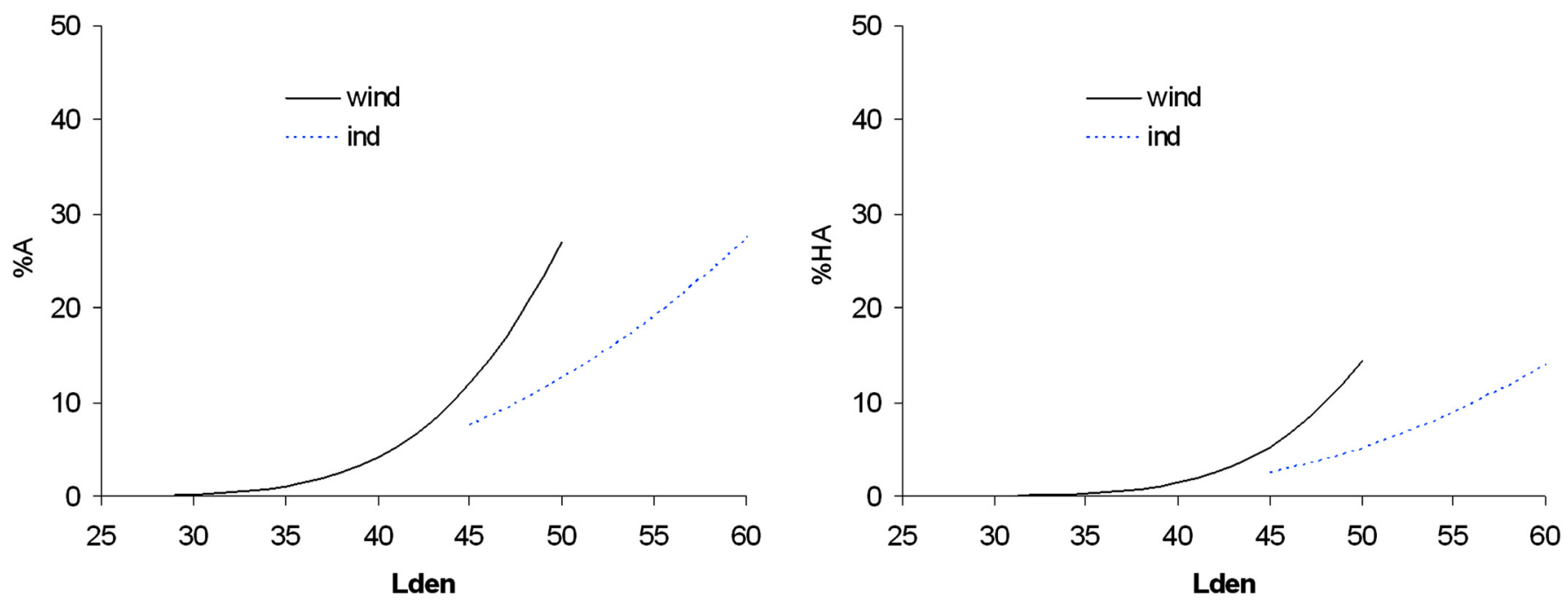

FIG. 2. (Color online) Comparison of the percentage of residents annoyed (\%A) or highly annoyed (\%HA) indoors due to wind turbine noise (wind) and industrial noise (ind).

highly annoyed (\%HA) persons indoors derived in the present study to the exposure-response relationships for industrial noise from stationary sources (Miedema and Vos, 2004) and (air, road, and rail) transportation noise (Miedema and Vos, 1998; Miedema and Oudshoorn, 2001). Note that while the polynomials for industrial noise may be extrapolated for the $L_{d e n}$ range $35-65 \mathrm{~dB}$, none of the respondents in the included studies were exposed to levels lower than $45 \mathrm{~dB}$ (Miedema and Vos, 2004). The database used for estimating exposure-response curves for transportation noise comprised $L_{d n}$ in the range between 42 and $75 \mathrm{~dB}$ (Miedema and Vos, 1998 ) which later was transformed into $L_{d e n}$ with approximately the same range (Miedema and Oudshoorn, 2001). An overview of the included transportation noise studies (Miedema and Vos, 1998) shows that, with the exception of aircraft noise studies, hardly any of them consider $L_{d n}$ levels below $50 \mathrm{~dB}$.

\section{DISCUSSION}

The present study shows that in comparison to other sources of noise, annoyance due to wind turbine noise is found at relatively low noise exposure levels. In the overlapping exposure range, the expected percentage of annoyed persons indoors by wind turbine noise is higher than that due to other stationary sources of industrial noise and also increases faster with increasing noise levels. Furthermore, the expected percentage of annoyed or highly annoyed persons due to wind turbine noise across the exposure range resembles the expected percentages due to each of the three modes of transportation noise at much higher exposure levels. However, some similarity is found in the range $L_{\text {den }}$ 40-45 dB between the percentage of annoyed persons by wind turbine noise and aircraft noise. Although the comparison may be hampered by differences between sources in
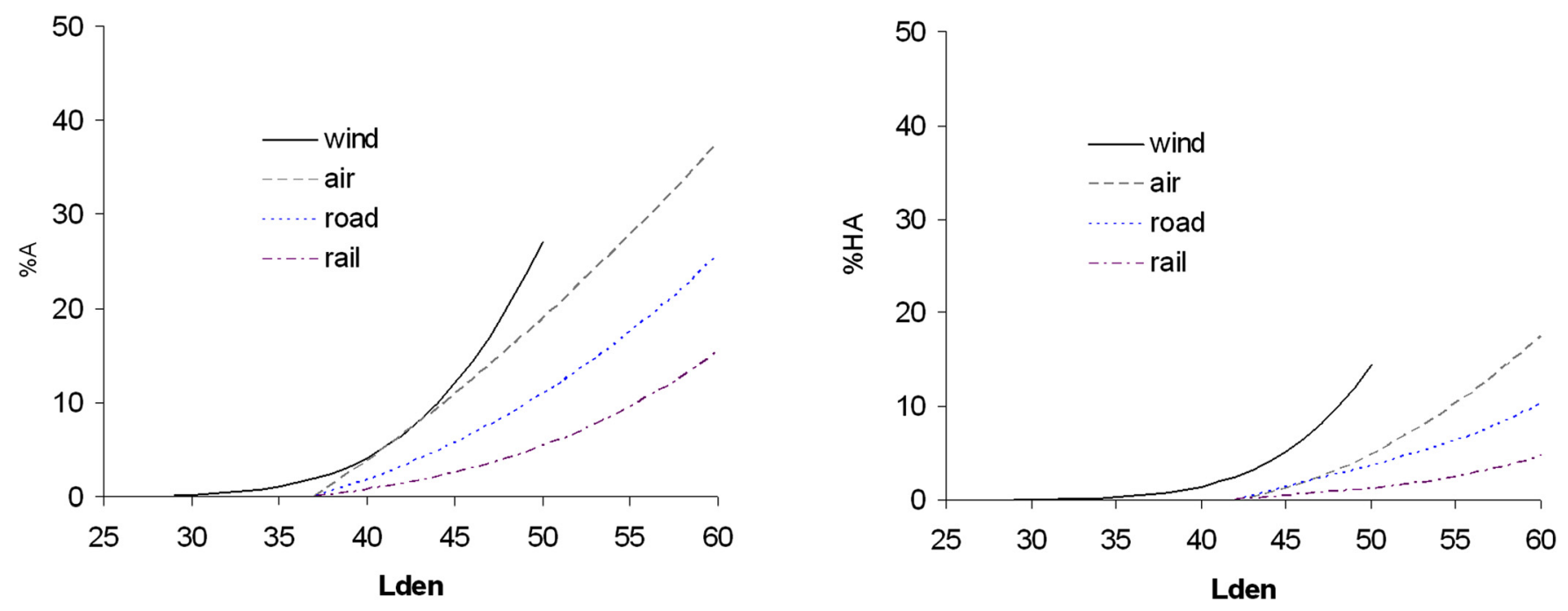

FIG. 3. (Color online) Comparison of the percentage of residents annoyed $(\% A)$ or highly annoyed $(\% H A)$ indoors due to wind turbine noise (wind) and due to transportation noise (air, road and rail). 
exposure range, and the confidence intervals at the high end of the wind turbine noise range are large, the results suggest that, at relatively low exposure levels, wind turbine noise induces an annoyance response that is expected to occur only at much higher levels of transportation noise and other industrial noise sources.

Besides noise exposure, various individual and situational characteristics were found to influence the level of annoyance: having economic benefit from the use of wind turbines or being able to see one or more wind turbines from within the home are two particularly influential situational factors; both of which have been reported to affect annoyance due to wind turbine noise before (Pedersen and Larsman, 2008; van den Berg et al., 2008). The economic benefit factor is reminiscent of earlier findings that being employed at the noise source (e.g., airport or industry) attenuates the annoyance reported (Miedema and Vos, 1999, 2004). Also, visibility from the home (e.g., living room, bedroom) has been reported earlier to affect annoyance from stationary sources (Miedema and Vos, 2004) and may exert its influence in different ways such as through visual intrusion, increased salience, or enhanced identification of the source of the noise. In addition, noise sensitivity and age had similar effects on annoyance to those found in research on annoyance by other sources. Noise sensitivity was found to be associated with increased annoyance, and, although for indoor noise annoyance only the linear component was significant, the overall form of the relationship between age and annoyance is similar to the inverse U-shaped relationship found for other noise sources (Miedema and Vos, 2004; Groothuis-Oudshoorn and Miedema, 2006). Hence response to wind turbine noise is influenced by similar situational and individual factors of which the strong influence of visibility on annoyance due to wind turbine noise may partly explain the unexpectedly high annoyance percentages.

Another factor that could possibly explain part of the relatively large annoyance response is the sound character of wind turbine noise. The noise is emitted from a level above the receiver, actually at several heights as the main source is the turbulence around the rotor blades at the outer part (Oerlemans et al., 2007); for modern wind turbines typically varying between 50 and $130 \mathrm{~m}$ over the ground as the rotor blades move. This gives an amplitude modulated sound, for example with an amplitude of $5 \mathrm{~dB}$ (van den Berg, 2009) and a modulation frequency of $0.5-1 \mathrm{~Hz}$. The sound power levels depend on the wind velocity, meaning that the immission levels also vary irregularly and unpredictably. Amplitude modulated sound is known to be easily perceived (Fastl and Zwicker, 2007), and a recent study suggests that the thresholds for wind turbine noise in the presence of natural sound are around -8 to $-12 \mathrm{~dB}$ signal-to-noise ratio (Bolin, 2009). This means that wind turbine sound may particularly be heard in otherwise quiet areas, where people do not expect to hear industrial noise.

Furthermore, the mostly rural position of wind turbines may contribute to the heightened annoyance response. In addition to the ambient noise level, the expectations of a living environment supposedly influence an individual's appraisal of an uncontrollable sound (Pedersen et al., 2007).
Studies on road traffic noise annoyance have mainly been carried out in urban areas, while wind turbines are situated in rural environments. The influence of area characteristics on annoyance due to wind turbine noise was not clear in this study. Differences in annoyance between rural and built-up areas reported previously (Pedersen and Larsman, 2008; van den Berg et al., 2008) were not found in the overall dataset, which might be the result of this variable showing opposing effects in the studies investigated. It is interesting that the study in the Netherlands only revealed an influence of background levels of road traffic noise on wind turbine annoyance when the level of wind turbine noise was moderate $\left[L_{d e n} 35-40 \mathrm{~dB}(\mathrm{~A})\right]$ and road traffic noise exceeded that level by at least $20 \mathrm{~dB}$ (Pedersen et al., 2010). However, all areas in these studies (rural or built-up) could be considered as rural or semi-rural in contrast to those explored in other community noise studies. Possibly there were some similarities between aircraft and wind turbine studies in the characteristics of study areas, as suburban areas with detached houses could surround an airport (see for example Lim et al., 2008), which could have manifested as some likeness between the exposure-response relationships for the two sources. Future studies should address the influence of the setting, not just in terms of differences in background levels, but also as a possible moderator in itself.

Differences in the perceived annoyance between the three studies on which the exposure-response relationship was based were not directly attributable to differences in individual or situational characteristics. However, the 2005 Swedish study did find lower levels of annoyance than either of the other studies and was conducted in areas that differed strongly in terms of the type of terrain compared to the other two studies. While the 2000 Swedish study and the study in the Netherlands were conducted in similar landscapes, i.e., flat terrain, half of the respondents of the 2005 Swedish study lived in a hilly area, which may have influenced not only the visibility of wind turbines from within the home, but the accurate assessment of the wind turbine noise exposure as well. Nevertheless, no significant differences between flat and complex terrain were found on the basis of the present dataset. Overall, indoor annoyance appeared to be lower in Sweden, which could be due to differences between the countries when it comes to tradition of building constructions, with highly insulated windows being more common in Sweden (i.e., triple window glass or double window glasses with argon).

Considering the large attenuating influence that economic benefit has on perceived annoyance and the possibly exaggerated number of individuals in the present sample who benefitted economically from the use of wind turbines, exposure-response relationships were derived only for respondents without economic interests in wind turbines. This approach differs from the derivation of other exposureresponse relationships, which did not exclude respondents who were employed at the noise source. In the present database, however, respondents with economical benefit lived primarily in the highest exposure categories and nevertheless hardly reported any annoyance, resulting in a drop in the annoyance response at the highest exposure levels only. 
Because of the regression method used, including these respondents would contaminate the exposure-response curve by unjustifiably pulling it downward over the whole range of exposure.

Another choice was to base the comparison with other noise sources on indoor noise annoyance. This makes for a conservative comparison because almost all respondents reported a higher level of annoyance outdoors than indoors, and the exposure-response relationships for other noise sources are largely based on studies that did not distinguish between annoyance indoors and outdoors. Because wind turbines are usually situated in rural or mildly built-up areas, annoyance outdoors might play a more important role than is the case for other noise sources because the residents of these areas may spend a greater proportion of their time outdoors. In order to limit annoyance due to wind turbine noise, policy makers should take into consideration both the expected annoyance indoors and outdoors.

The proposed exposure-response relationships for annoyance by wind turbine noise are only based on three studies, and more studies are undeniably needed. Still, they may already serve as indicative for suitable regulations or for the evaluation of existing legislation. For example, regions with a highest allowed immission level of $45 \mathrm{~dB}(\mathrm{~A})$ equivalent level [corresponding to $L_{d e n} 49.7 \mathrm{~dB}(\mathrm{~A})$ in this study] such as Denmark could expect less than $14 \%$ of the exposed population to be highly annoyed indoors by wind turbines and less than $29 \%$ to be highly annoyed outdoors. However, it should be noted that situational factors, as well as possible cultural differences, may lead to considerable deviation from the curve in specific cases.

\section{ACKNOWLEDGMENTS}

The authors would like to thank Dr. Frits van den Berg of the Amsterdam Public Health Service (formerly of the University of Groningen) for his much valued advice and for making his research data available to the authors. The present study was funded by the Ministry of Housing, Spatial Planning, and the Environment (VROM) of the Netherlands.

Bolin, K. (2009). "Wind turbine noise and natural sounds-making, propagation and modeling," Ph.D. thesis, Royal Institute of Technology, Sweden.

Fastl, H., and Zwicker, E., (2007). Psychoacoustics: Facts and Models (Springer-Verlag, New York), Chap. 10, pp. 247-256.
Groothuis-Oudshoorn, C. G. M., and Miedema, H. M. E. (2006). "Multilevel grouped regression for analyzing self-reported health in relation to environmental factors: The model and its application,” Biom. J. 48, 67-82.

ISO 1996-2: 2002, Acoustics: Description, measurement, and assessment of environmental noise. II. Determination of environmental noise levels (International Organization for Standardization, Geneva, Switzerland, 2002).

Lim, C., Kim, J., Hong, J., and Lee, S. (2008). "Effect of background noise levels on community annoyance from aircraft noise," J. Acoust. Soc. Am. 123, 766-771.

Miedema, H. M. E., and Oudshoorn, C. G. M. (2001). "Annoyance from transportation noise: Relationships with exposure metrics DNL and DENL and their confidence intervals," Environ. Health Persp. 109, 409-416.

Miedema, H. M. E., and Vos, H. (1998). "Exposure-response relationships for transportation noise," J. Acoust. Soc. Am. 104, 3432-3445.

Miedema, H. M. E., and Vos, H. (2004). "Noise annoyance from stationary sources: relationships with exposure metric day-evening-night level (DENL) and their confidence intervals," J. Acoust. Soc. Am. 116, 334-343.

Oerlemans, S., Sijtsma, P., and Méndez López, B. (2007). "Location and quantification of noise sources on a wind turbine," J. Sound Vibr. 299, 869-883.

Pedersen, E. (2011). "Health aspects associated with wind turbine noise Results from three field studies," Noise Control Eng. J. 59, 47-53.

Pedersen, E., Hallberg, L. R.-M., and Persson Waye, K. (2007). "Living in the vicinity of wind turbines - a grounded theory study," Qual. Res. Psych. 4, 49-63.

Pedersen, E., and Larsman, P. (2008). "The impact of visual factors on noise annoyance among people living in the vicinity of wind turbines," J. Environ. Psychol. 28, 379-389.

Pedersen, E., and Persson Waye, K. (2004). "Perception and annoyance due to wind turbine noise - a dose-response relationship," J. Acoust. Soc. Am. 116, 3460-3470.

Pedersen, E., and Persson Waye, K. (2007). "Wind turbine noise, annoyance and self-reported health and well-being in different living environments," Occ. Environ. Med. 64, 480-486.

Pedersen, E., and Persson Waye, K. (2008). "Wind turbines-low level noise sources interfering with restoration?" Environ. Res. Lett. 3, 1-5.

Pedersen, E., van den Berg, F., Bakker, R., and Bouma. J. (2009). "Response to noise from modern wind farms in The Netherlands," J. Acoust. Soc. Am. 126, 634-643.

Pedersen, E. van den Berg, F., Bakker, R., Bouma, J. (2010). "Can road traffic mask sound from wind turbines? Response to wind turbine sound at different levels of road traffic sound," Energy Policy. 38, 2520-2527.

van den Berg, F. (2008). "Criteria for wind farm noise: Lmax and Lden," in Proceedings of the 7th European Conference on Noise Control, EURONOISE, Acoustics'”08, Paris, France.

van den Berg, F. (2009). "Why is wind turbine noise noisier than other noise?" in Proceedings of the 8th European Conference on Noise Control, EURONOISE, Edinburgh, United Kingdom.

van den Berg, F., Pedersen, E., Bouma, J., Bakker, R. (2008). "Windfarm perception: Visual and acoustic impact of wind turbine of wind turbine farms on residents," Report FP6-2005-Science-and-Society-20. University of Groningen, Groningen, The Netherlands and University of Gothenburg, Gothenburg, Sweden. 